$15^{\text {th }}$ International Conference on

AEROSPACE SCIENCES \& AVIATION TECHNOLOGY,

$\boldsymbol{A S A T}$ - 15 - May 28 - 30, 2013, Email: asat@mtc.edu.eg,

Military Technical College, Kobry Elkobbah, Cairo, Egypt,

Tel: +(202) 24025292-24036138, Fax: +(202) 22621908

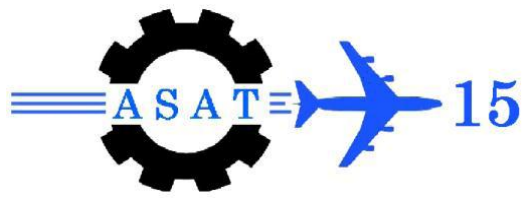

\title{
Fabrication and Characterization of Carbon Nanotubes/PMMA Nanocomposite
}

\author{
$\{\text { Eman A. Mwafy*, S. S. A. Obayya }\}^{\dagger}$
}

\begin{abstract}
The physical/chemical properties of carbon nanotube (CNT) have attracted much interest for applications in different fields, from microelectronic to photonic technology, due to their exiting conductivity properties, high hardness and high resistance to thermal stress. The technology to produce CNT with the desired properties is still under development. In this work, we report on fabrication CNT deposited by simple chemical route via the reaction between ferrocene and ammonium chloride in air at low temperature. UV-VIS absorption spectroscopy and Transmission Electron Microscopy (TEM) provide the experimental confirmation that CNT-like structures are present then our intention to get nanocomposites consist of CNTs and a poly methyl methacrylate by melt mixing process with high mechanical properties for principal concepts of the preparation of such materials for different applications.
\end{abstract}

Keywords: Carbon nanotube (CNT); nanocomposites; functionalization

\section{Introduction}

\subsection{Carbon Nanotubes}

Since carbon nanotubes (CNTs) were reported by Iijima in 1991, they have attracted worldwide interest and have become one of the most promising building blocks for nanotechnology because of their unique structures and excellent mechanical, chemical and electronic properties [1]. However, most papers in this field focus on crystalline CNTs with perfect concentric graphene layers such as single wall CNTs and multiwall CNTs. In fact, amorphous carbon nanotubes (a-CNTs), whose walls have amorphous structure due to the defects in the carbon network, are expected to lead to more interesting properties and thus the development of new potential nanodevices. For example, they could be used as gaseous adsorbent and catalyst support with high efficiency because of their porous structure and chemical inertnes [2]; also can be used in nanoelectronics and sensor devices because of their absence of chirality problem as well as larger semiconductor band gap which is inversely proportional to the diameter compared with the crystalline nanotubes [3].

For these reasons, scientists have paid much more attention to the CNTs in recent years. Numerous methods for the synthesis of CNTs have been developed [3-5]. Among the variety of methods, chemical vapor deposition (CVD) is the most widely used to synthesize CNTs.

emwafy@zewailcity.edu.eg.

$\dagger$ Center for Photonics and Smart Materials (CPSM), Zewail City of Science and Technology, Sheikh Zayed District, 6th of October city, Giza, Egypt. 
Although CVD offers the benefit of significantly lower synthesis temperatures than arcdischarge and laser ablation techniques, it still requires a growth temperature of 600-950 C. For both of the methods, however, catalysts are essential as usual but the synthesis temperature is still relatively high $[6,7,8]$. Additionally, for all the above methods, oxygen should be avoided during the whole process to prevent the CNTs from being oxidized so scientists directed their aim to get a simple chemical route for synthesis CNTs at low temperatures.

\subsection{CNT Based Polymer Composites}

Polymeric nanocomposites (PNCs) or polymer nanostructured materials represent a radical alternative to conventional filled polymers. The reinforcement of polymers is done by fillers, which play a major role in strengthening the composites.

One of the most intriguing applications of CNT is the polymer/CNT nanocomposites including transportation, automotive, aerospace, defence, sporting goods, energy and infrastructure sectors. This is due to their high durability, high strength, light weight, design and process flexibility, etc. [8-13]. The high mechanical (exceptionally high tensile strength and stiffness) [14], electrical and thermal properties of CNT make them ideal candidates as fillers in light weight polymer composites.

Polymers such as epoxy [10], thermoplastics [11], gels[12], as well as poly (methyl methacrylate) (PMMA)[9] have been used as the matrix. The conductivity, strength, elasticity, toughness, and durability of formed composites may all be substantially improved by the addition of nanotubes $[15,16]$.

However, the effective utilization of carbon nanotubes in composite applications depends strongly on the ability to homogeneously disperse them throughout the matrix without destroying their integrity. Furthermore, good interfacial bonding is required to achieve load transfer across the CNT-matrix interface, a necessary condition for improving the mechanical properties of the composite [17]. Load transfer from matrix to CNTs plays a key role in the mechanical properties of composites. If the adhesion between the matrix and the CNTs is not strong enough to sustain high loads, the benefits of the high tensile strength of CNTs are lost. Load transfer depends on the interfacial shear stress between the filler and the matrix [18].

In this paper, thus, a simple chemical route has been developed to synthesize a-CNTs via the reaction between ferrocene and ammonium chloride in air at only at low temperature. Then carbon nanotubes treated and reinforced in PMMA to form enhanced mechanical polymer nanocomposites.

\section{Materials and Experimental}

All reagents used were analytically pure from Sigma Aldrich with purity of $99.9 \%$.

\subsection{Preparations of CNTs}

In a typical experiment process [19], Two $\mathrm{g}$ of ferrocene and $4 \mathrm{~g}$ of ammonium chloride fine powders were first mixed and placed in a quartz boat. The boat was covered to prevent ferrocene from escaping rapidly as a result of its sublimation. Then, the quartz boat was put into an air furnace and heated to $250{ }^{\circ} \mathrm{C}$. After being held for one hour, the furnace was allowed to cool down naturally and a black powder was found in the boat. The powder was washed with $\mathrm{HCl}$ solution and de-ionized water in sequence for several times, and then dried in an oven at $50^{\circ} \mathrm{C}$ for $24 \mathrm{~h}$. Finally small amount of black powder was obtained totally. 


\subsection{Treatment of CNTs}

Due to the low reactivity and hydrophobitty of carbon nantube surface, introduction of reactive groups via treatment of CNTs by silane (3-methacyloxypropyl trimethoxy silane), (KH570) is very important step.

The introduction of reactive groups onto the surface of CNTs was achieved through the reaction between silane KH570 as follow: $2.0 \mathrm{~g}$ CNTs and $2.0 \mathrm{ml} \mathrm{KH570} \mathrm{in} \mathrm{40ml} \mathrm{o-xylene}$ were kept at $150^{\circ} \mathrm{C}$ for $3 \mathrm{~h}$ under stirring and $\mathrm{N} 2$ protection. After that, the CNTs were collected by filtration and rinsed three times with acetone. Afterwards, the treated CNTs were dried under vacuum for $24 \mathrm{~h}$ at $100^{\circ} \mathrm{C}$.

\subsection{Preparation of Nanofluid}

A good dispersion not only makes more filler surface area available, but also prevents aggregation of the filler. The chemically treated $2 \mathrm{gm}$ of CNTs were mixed with $500 \mathrm{ml}$ of distilled water for making the $0.1 \mathrm{wt} \%$ of CNT/PMMA specimen similarly for making $0.3 \mathrm{wt}$ of CNT/PMMA $6 \mathrm{gm}$ of CNTs are mixed with $500 \mathrm{ml}$ of water and for $0.5 \mathrm{wt} \%$ of CNT/PMMA $10 \mathrm{gm}$ of CNTs were mixed with $500 \mathrm{ml}$ of distilled water. After that each nanofluid was then sonicated for one hour in a bath sonicator to have a homogenous dispersion of CNTs with solvent. The suspension can later be mixed with the polymer and the solvent can simply be evaporated by heating [20,21].

\subsection{Preparation of CNT- PMMA Nanocomposites}

Nanocomposites were made by melt blending PMMA pellets with a given ratio of MWNTs (between 0.1 and $1 \mathrm{wt} \%$ ). This preblend was then fed into mixing head with a volumetric capacity of $69 \mathrm{~cm} 3$. The blends were processed for $10 \mathrm{~min}$ at a twin-screw extruder (Type: DSM Xplore) (model 600) with roller blades and a $100 \mathrm{rpm}$ and $230{ }^{\circ} \mathrm{C}$ using a counter rotating screw configuration.

\subsection{Preparation of Specimen}

CNT / PMMA mix were used as a raw material in an injection moulding machine. PMMA was melted at the plasticized unit of the injection moulding machine which was kept at $200 \mathrm{C}$ to induce sufficient softening of polymer to mix with CNTs and this mixture injected into a tensile specimen. The test specimen has the basic shape of a tensile $4 \mathrm{~mm}$ thick and $50 \mathrm{~mm}$ long.

\section{Results and Discussion}

Morphology and physical properties of carbon nanotubes and well dispersed CNTs/PMMA composites have been analyzed

Transmission electron Microscope (TEM)(JEOL-JEM-1011, Japan) shows in Fig. 1 that a typical TEM image of one single carbon nanotube is provided and the dark area in the image corresponds to the wall of the nanotube. it is apparent that the walls of the CNTs are rough, indicating that the nanotubes have many defects, which is attributable to the low synthesis temperature also showing that is an MWCNT appeared as an dark lines also showed that . The a-CNTs are about $200 \mathrm{~nm}$ in diameter and $10-18 \mu \mathrm{m}$ in length.

Double beam spectrophotometer (T80+ UV-vis instrument, PG Instrument Ltd.) to measure absorbance as shows in Fig. 2 that an absorption maximum between 200 and $300 \mathrm{~nm}$, UV range, is observed, indicating dispersion of individual MWCNTs taking into account that larger surface area of MWCNT leads to higher absorbance, it would be expected that a more efficient dispersion will produce an increment in absorption. 


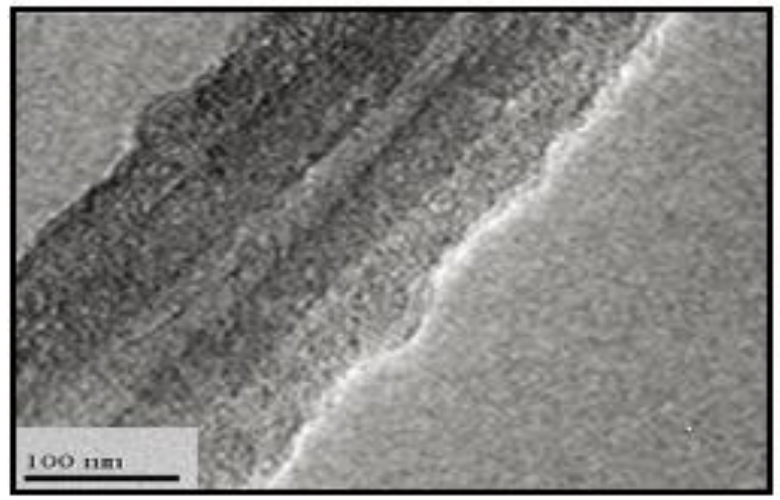

Fig.1 TEM images of one single CNT

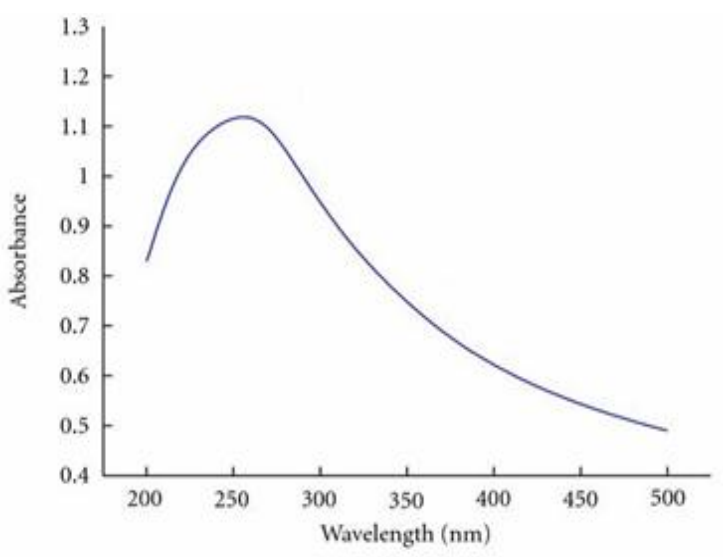

Fig. 2 UV-vis spectrum of MWCNT

Figure 3 shows the SEM images of the composites indicating that well-dispersed MWCNT preblend with PMMA is dispersed homogenously in the matrix while there are remaining portions of agglomerates appeared at relatively high concentration (1\% CNT).

Sheets were cut out to five individual strips specimens by a steel die of constant width $(0.4$ $\mathrm{cm})$. The thickness of the test specimen was determined by using a dial gauge. A bench mark of $25 \mathrm{~mm}$ length was marked on the working part of each specimen under test. Mechanical tests including tensile strength, and elastic modulus were performed at room temperature $\left(23 \pm 2{ }^{\circ} \mathrm{C}\right)$ using a tensile testing machine (Model Z 010, Zwick, Germany) at a crosshead speed of $50 \mathrm{~mm} / \mathrm{min}$, according to ASTM D-638 standards.

Figures 4 and 5 showed that both the Elastic modulus and the tensile strength significantly increase upon adding small amounts of MWNT, thus confirming earlier data [10-12]. A 25\% increase in tensile strength and Young's modulus of PMMA reinforced with only $0.5 \mathrm{wt} \%$ of MWCNT. The f-MWNT based composite studied here exhibits better mechanical performance, which may be attributed to the quality of the nanotube dispersion in the matrix and the better tube-matrix interfacial adhesion but higher loadings of CNTs in a composite do not perform as well as lower loadings suggesting an increase in voids or other defects as the loading of CNTs increases, likely due to the difficulty of homogeneously dispersing concentrated CNT/polymer melts or solutions. They reduce reinforcing effects of CNTs and result in a lower tensile strength. A reduction in the agglomerate size can be achieved with a functionalization process that the functional groups, which develop on the surfaces of the 
CNTs, lead to steric hindering electrostatic interactions resulting in a better distribution of CNTs in the matrix.

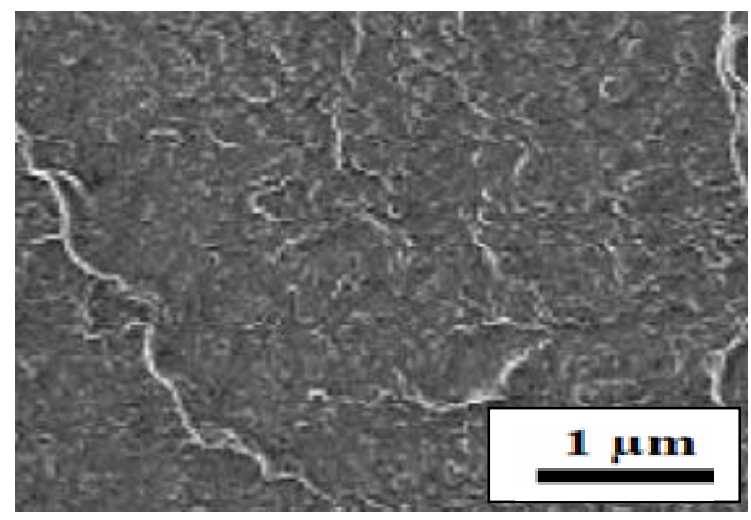

a

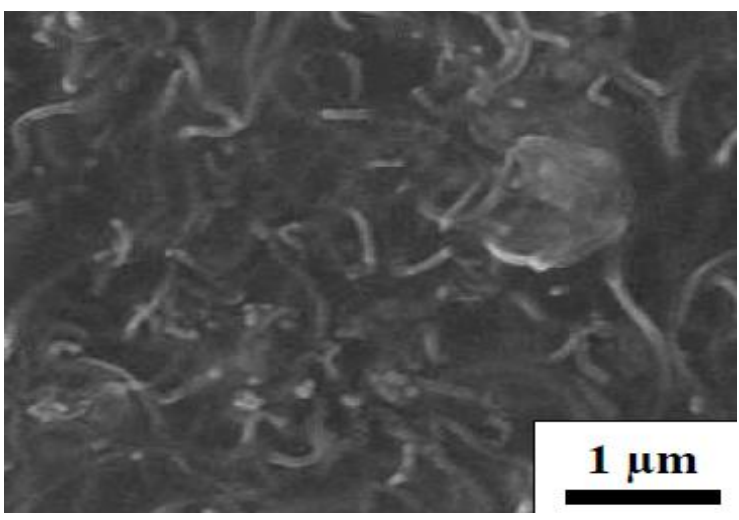

b

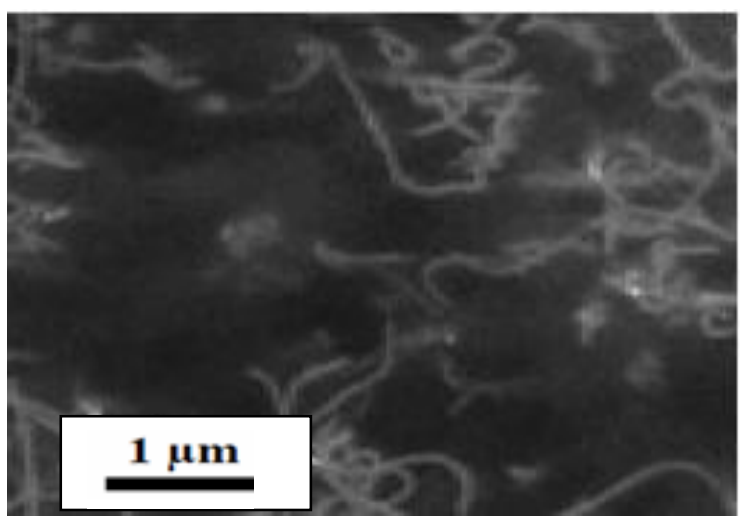

$\mathrm{c}$

Fig. 3: SEM images for the fracture of well-dispersed MWCNT /PMMA composites with different wt fraction of CNT (a) $0.1 \%$ CNT, (b) $0.5 \%$ CNT and (c)1 \% CNT .

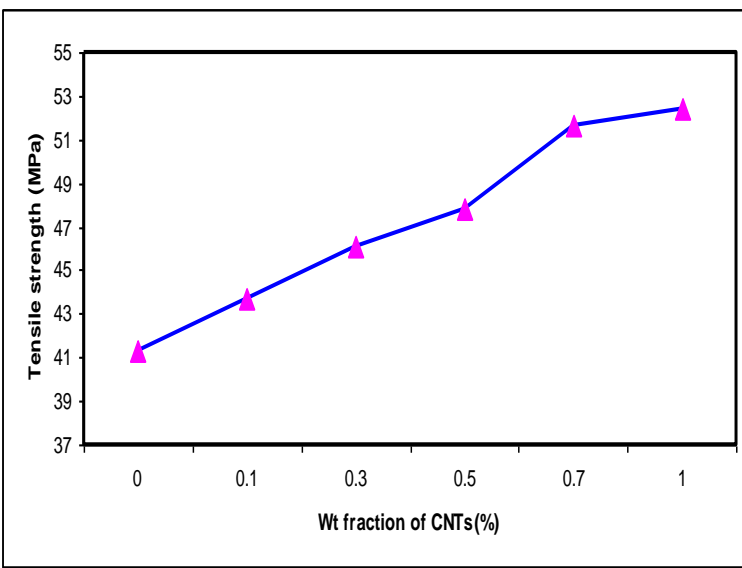

Fig. 4: Tensile strength of MWCNT /PMMA composites with different wt fraction of CNT 


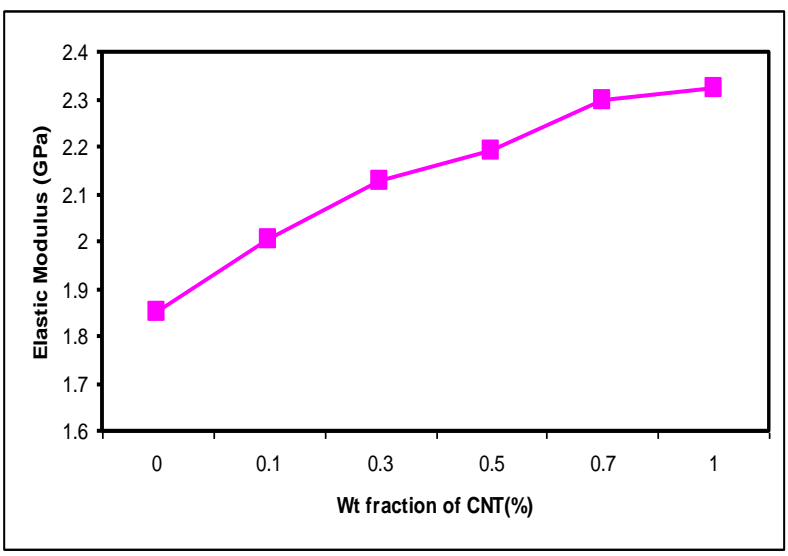

Fig. 5: Elastic modulus of MWCNT /PMMA composites with different wt fraction of CNT

The hardness test was performed according to ASTM D 2240 standards using hardness tester (Zwick 3150, Germany). Fig.6 shows the average of five different measurements distributed over the specimen was taken which indicate that hardness increases with increase in CNTs content.

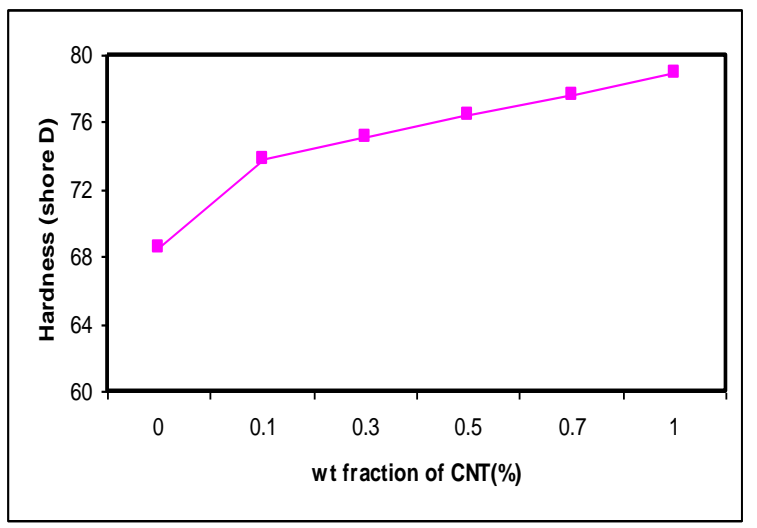

\section{Fig. 6: Hardness of MWCNT /PMMA composites} with different wt fraction of CNT

This may be explained in terms of the homogensity of dispersion between PMMA and CNTs then hardness decrease due to agglomeration of tubes forming voids or weak points inside the composite. These results obtained were found to be similar to those for the tensile strength, i.e. as the filler content increases, the hardness increases.

\section{Conclusion}

In summary, we have developed a simple chemical route to prepare a-CNTs with open ends and uniform diameters via the reaction between ferrocene and ammonium chloride at $250 \mathrm{C}$ in air. The oxygen in air surprisingly acts as a reactant in the method and the formation of aCNTs is regarded as a self-assembly process. The a-CNTs are about $200 \mathrm{~nm}$ in diameter and $10-18 \mu \mathrm{m}$ in length, respectively. Production of this kind of a-CNTs under such amoderate synthesis condition is attractive for diverse applications in nanoelectronics and sensors in the future. Incorporating low loadings of MWCNTs (0.1, 0.3, 0.5 and $0.7 \mathrm{wt} \%)$ to PMMA improved the mechanical properties of the resultant nanocomposite. Higher loadings (1wt\%) 
provided lesser improvements in the mechanical properties, and in some cases significant reductions were recorded. The extent of the effect was dictated by the chemical functional groups added to the MWCNT, and the level of loading used. Improvements in mechanical properties were attributed to the MWCNTs being well dispersed within the PMMA. The dispersion of MWCNTs within the matrix is enhanced by adding chemical functional groups, with the carboxyl functionalized MWCNTs providing the most significant improvements in mechanical integrity.

\section{Acknowledgement}

The authors acknowledge the financial support of Center for Photonics and Smart Materials (CPSM), Zewail City of Science and Technology.

\section{References}

[1] Iijima S. Helical microtubes of graphitic carbon. Nature 1991; 354(6348):56-8.

[2] Poncharal P, Wang ZL, Ugarte D, Heer WAD.Electrostatic deflections and electromechanical resonances of carbon nanotubes. Science 1999;283 (5407):1513-6

[3] Rakitin A, Papadopoulos C, Xu JM.Electronic properties of amorphous carbon nanotubes. Phys Rev B 2000;61(8):5793-6.

[4] Liu YN, Song XL, Zhao TK, Zhu JW, Hirscher M, Philipp F. Amorphous carbon nanotubes produced by a temperature controlled DC arc discharge. Carbon 2004;42(89):1852-5.

[5] Zhao NQ, He CN, Du XW, Shi CS, Li JJ, Cui L. Amorphous carbon nanotubes fabricated by low-temperature chemical vapor deposition. Carbon 2006;44(9):1859-62.

[6] Chen JT, Shin K, Leiston-Belanger JM, Zhang MF, Russell TP. Amorphous carbon nanotubes with tunable properties via template wetting. Adv Funct Mater 2006;16(11):1476-80.

[7] Nishino H, Nishida R, Matsui T, Kawase N, Mochida I. Growth of amorphous carbon nanotube from poly(tetrafluoroethylene) and ferrous chloride. Carbon 2003;41(14):2819-23.

[8] Hersam M C, Hoole A C F, O’Shea S J and Welland M E 1998 Appl. Phys. Lett. 72915

[9] Laurie O, Cox D E and Wagner H D 1998 Appl. Phys. Lett. 81- 1638

[10] Ruiz Facundo, Sun W D, Pollak Fred H and Venkatraman Chandra 1998 Appl. Phys. Lett. 73-1802

[11] Fiege G B M, Altes A, Heiderhoff R and Balk L J 1999 J. Phys D32-L13

[12] Gomes S, Trannoy N and Grossel P 1999 Meas. Sci. Technol 10-805

[13] Cai L, Tabata H and Kawai T 2000 Appl. Phys. Lett. 77-3105

[14] Wong E W, Sheehan P E and Lieber C M 1997 Science 277 -1971

[15] Grimes C A, Mungle C, Kouzoudis D, Fang S and Eklund P C r...Chem. Phys. Lett. $319-460$

[16] Grimes C A, Dickey E C, Mungle C, Ong K G and Qian D 2001 J. Appl. Phys. 90-4134

[17] Andrew Rodney, Jacques David, Qian Dali and Rantell Terry 2002 Acc. Chem. Res. 35-1008

[18] Schadler L S, Giannaris S C and Ajayan P M 1998 Appl. Phys . Lett. 73-3842

[19] Xiong YJ, Xie Y, Li XX, Li ZQ.Production of novel amorphous carbon nanostructures from ferrocene in low-temperature solution. Carbon 2004;42(8-9):1447-53.

[20] Liu Luqi and Wagner H D 2005 Composites Sci. \& Technol. 65-1861

[21] Lu K L, Lago M, Chen Y K, Green M L H, Harris P J F and Tsang S C 1996 Carbon 34-814 